\title{
Nématodes (Trichostrongyloidea) parasites d'un Marsupial de Guyane
}

\author{
par Marie-Claude DURETTE-DESSET \\ Laboratoire de Zoologie (Vers) associé au C.N.R.S. ( $\mathrm{P}^{r}$ A.-G. Chabaud) \\ Muséum national d'Histoire naturelle, 43, rue Cuvier, F 75231 Paris Cedex 05
}

\section{Résumé.}

L'autopsie d'un Metachirops opossum (Seba) originaire de Guyane française, a permis de récolter quatre espèces de Trichostrongyloidea dont deux nouvelles: Viannaia viannai Travassos, 1914, Moennigia sp, Travassostrongylus paraquintus n. sp. et Viannaia metachirops $\mathrm{n}$. sp.

Les deux espèces dominantes ( $T$. paraquintus et $V$. viannai) sont réparties de façon sensiblement équivalente dans l'intestin. Cette répartition uniforme est différente de celle qui existe lorsque les espèces sont congénères. Dans ce dernier cas, il existe des niches écologiques bien précises que l'on ne retrouve pas ici.

La présence dans l'intestin de quatrièmes stades larvaires appartenant aux genres Viannaia, Moennigia et Travassostrongylus nous a permis d'en connaître les synlophes.

L'étude de celui du genre Travassostrongylus confirme deux points importants sur l'évolution des Héligmosomes: a) l'appartenance de ce genre aux Molineinae; b) la présence chez l'adulte d'arêtes dorsales, absentes chez la larve, apporte une preuve ontogénique aux hypothèses formulées précédemment, selon lesquelles le genre Viannella dériverait du genre Viannaia et le genre Longistriata du genre Suncinema.

\section{Summary.}

Nematodes (Trichostrongyloidea) parasites of a Marsupial from french Guyana.

« The autopsy of a Metachirops opossum (Seba) from french Guyana has enabled the author to recover four species of Trichostrongyloidea, two of them are new species.

Both the dominant species (Travassostrongylus paraquintus et Viannaia viannai) are evenly distributed along the digestive tract; thus showing a pattern of distribution differing from what is usually observed when several species of the same genus occur in the same host, and are to be found into well-delimited ecological niches. 
The fourth larval stages of the following genera Viannaia, Moennigia and Travassostrongylus were found in the host intestine, thus allowing for the study of their larval synlophes.

The study of Travassostrongylus synlophe has confirmed two important facts in the problem of Heligmosomidae evolution: firstly the validity of the placement of Travassostrongylus within the family Molineinae is reinforced, secondly the fact that cuticular dorsal ridges occur in the adult and are lacking in the larvae brings another ontogenic argument in support of the previously set up hypothesis, i. e. the genus Viannella should have originated from the genus Viannaia and the genus Longistriata from the genus Suncinema.

Lors d'une mission en Guyane Française en 1971, le $\mathrm{P}^{\mathrm{r}}$ A. G. Chabaud a récolté à notre intention les Trichostrongyloidea parasites de divers Marsupiaux.

Cette première note a pour objet l'étude des parasites de l'intestin d'un Metachirops opossum (Seba). Après division de l'intestin en trois parties sensiblement égales, tous les helminthes ont été récoltés et comptés. Parmi les animaux récoltés, se trouvent des quatrièmes stades larvaires des genres Viannaia Travassos, 1914, Moennigia Travassos, 1935 et Travassostrongylus Orloff, 1933, dont l'étude se révèle intéressante en ce qui concerne la phylogenie des Trichostrongyloidea en général. Nous traiterons ce sujet à la fin de l'article.

\section{1) Étude des espèces :}

Travassostrongylus paraquintus n. sp.

MAtÉRIEL : 27 б, 22 \&, 4 stades IV.

Localisation : $1 / 3$ antérieur de l'intestin $=10$ б, 7 \&, 4 stades IV ; $2 / 3=12$ б, $7 q ; 1 / 3$ postérieur $=5 \delta$, 8 ㅇ.

Description: Petits Nématodes légèrement recourbés le long de leur ligne ventrale. En vue apicale, la tête porte deux amphides, quatre papilles céphaliques externes, six papilles labiales externes. La bouche est entourée d'un anneau chitinoïde de forme triangulaire (fig. 1, B). Vésicule céphalique asymétrique, plus haute du côté dorsal que du côté ventral. Pore excréteur situé nettement en arrière de la fin de l'œsophage. Deirides arrondies mais portant une fine pointe en leur milieu, situées au même niveau

FIG. 1. - Travassostrongylus paraquintus n.sp. $\uparrow-\mathrm{A})$ extrémité antérieure, vue latérale gauche; B) tête en vue apicale; C) extrémité postérieure, vue latérale droite; D) région des ovéjecteurs, vue latérale droite; E) coupe transversale au milieu du corps; F) niveau du pore excréteur et des deirides, vue ventrale; - $\left.\delta^{*}-\mathrm{G}\right)$ bourse caudale, vue ventrale; $\mathrm{H})$ côtes dorsale et externo-dorsale, vue dorsale; I cône génital, vue ventrale; $\mathrm{J}, \mathrm{K})$ spicule gauche disséqué, successivement, vue latérale et vue dorsale; $\mathrm{L})$ ensemble cône génital et côte dorsale, vue latérale gauche; M) gubernaculum, vue latérale droite. A, G, J, K,: éch. $100 \mu$; B, C, D, E, F, H, I, L, M: éch. $50 \mu$. 


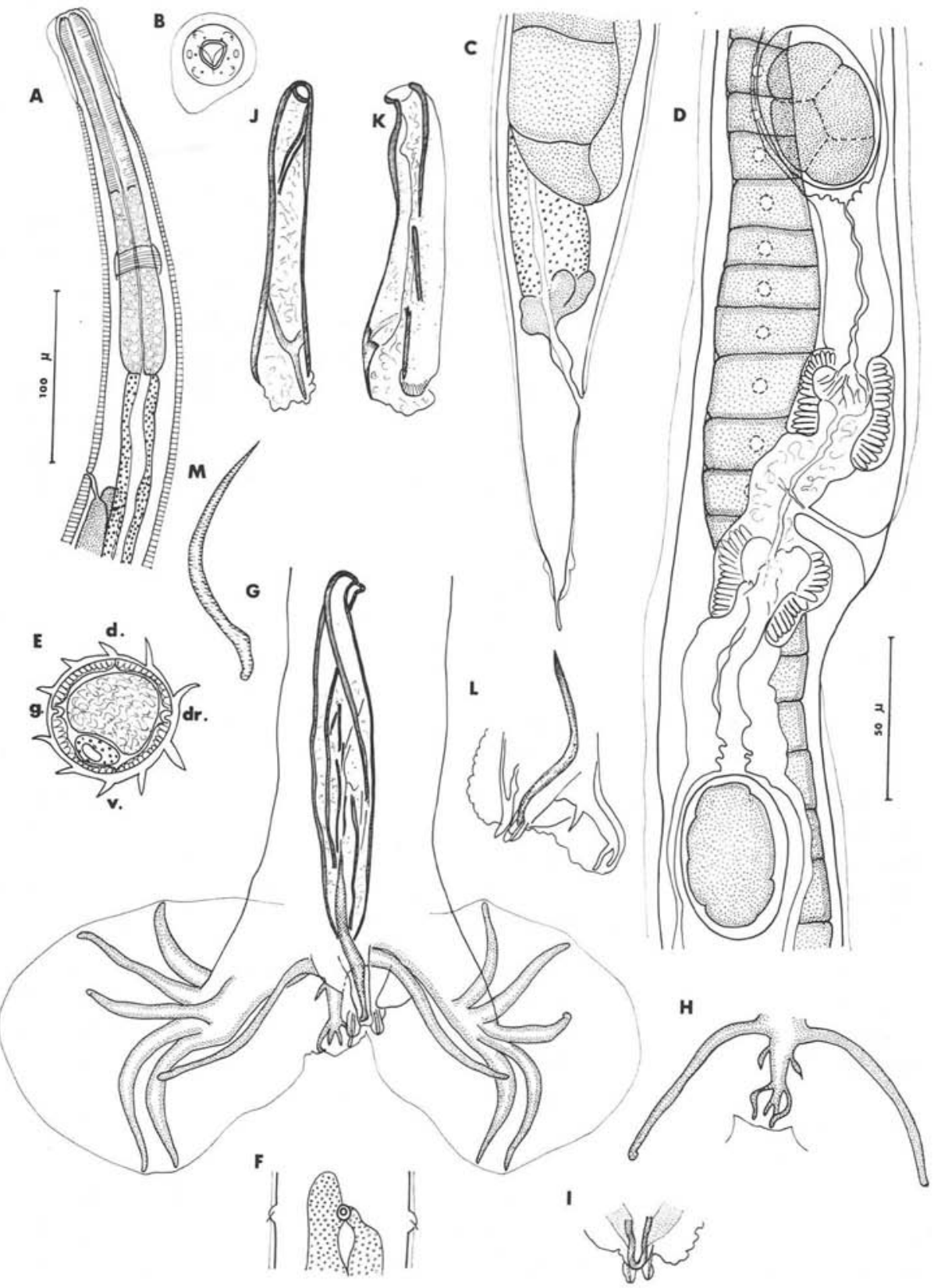

Annales de Parasitologie humaine et comparée (Paris), t. 49, $\mathrm{n}^{\circ} 5$. 
que l'anneau nerveux (fig. 1, F). Séparation entre œsophage musculaire et glandulaire nette. Glandes excrétrices bien visibles.

Synlophe: De type Travassostrongylus avec dix crêtes cuticulaires, cinq dorsales, cinq ventrales, orientées de la gauche vers la droite, symétriques par rapport à l'axe frontal (fig. 1, E).

Male : Corps long de $2,45 \mathrm{~mm}$, large de $50 \mu$ dans sa partie moyenne. Vésicule céphalique haute de $60 \mu$ (côté dorsal), $55 \mu$ (côté ventral) sur $25 \mu$ de large. Anneau nerveux, pore excréteur et deirides situés respectivement à $145 \mu, 265 \mu$ et $267 \mu$ de l'apex. Esophage long de $215 \mu$ (107 $\mu$ œsophage musculaire, $108 \mu$ œsophage glandulaire). Le testicule débute à $300 \mu$ en arrière de la fin de l'œsophage.

Bourse caudale subsymétrique avec un lobe droit plus développé. Lobes latéraux très développés. Lobe dorsal très petit. Côtes 8 longues et fines naissant à la racine de la côte dorsale. Celle-ci est divisée à son extrémité distale en quatre rameaux dont les médians sont les plus courts. Elle porte juste en arrière de la naissance des côtes 8 deux rameaux qui peuvent peut-être être interprétés comme étant les côtes 9 (fig. 1, H, L).

Spicules subégaux, ailés, hauts de $130 \mu$. Leur extrémité distale est enfermée dans une membrane soutenue par trois branches (fig. 1, J, K).

Le gubernaculum, haut de $70 \mu$, est fortement coudé du côté dorsal, ce qui lui donne de profil la forme d'une faucille (fig. 1, M).

Cône génital bien marqué portant sur sa lèvre antérieure la papille zéro et sur sa lèvre postérieure les deux papilles 7 en forme de pétale. La lèvre antérieure est chitinisée sur la moitié de sa hauteur (fig. 1, I).

Femelle: Corps long de $3 \mathrm{~mm}$, large de $50 \mu$ dans sa partie moyenne. Vésicule céphalique haute de $62 \mu$ (côté dorsal), $52 \mu$ (côté ventral) sur $26 \mu$ de large. Anneau nerveux, pore excréteur et deirides situés respectivement à $140 \mu, 280 \mu$ et $282 \mu$ de l'apex. Esophage long de $215 \mu(107 \mu$ œsophage musculaire, $108 \mu$ œsophage glandulaire). L'ovaire antérieur débute à $200 \mu$ en arrière de la fin de l'œsophage (fig. 1, A).

Didelphie. La vulve s'ouvre à $450 \mu$ en avant de la pointe caudale. Vagina vera long de $28 \mu$. La branche antérieure de l'ovéjecteur mesure $100 \mu$ (vestibule $20 \mu$, sphincter $30 \mu$, trompe $50 \mu$ ) ; la branche postérieure mesure $87 \mu$ (vestibule $10 \mu$, sphincter $32 \mu$, trompe $45 \mu$ ). Les deux branches utérines antérieure et postérieure mesurent respectivement $310 \mu$ et $210 \mu$, et contiennent 5 œufs pour la première, 4 œufs pour la seconde. Les œufs sont hauts de $50 \mu \times 35 \mu$, non segmentés (fig. 1, D).

Queue longue de $70 \mu$, portant une fine pointe de $10 \mu$ (fig. 1, C).

Discussion: Les spécimens du Metachirops présentent de grandes affinités avec T. quintus Freitas, 1937, parasite de Metachirus nudicaudatus personatus dans l'Etat de Rio au Brésil : la disposition des côtes bursales est la même ainsi que la forme du gubernaculum; de plus, chez les deux espèces, les spicules présentent trois rameaux terminaux. Cependant, un certain nombre de caractères diffèrent. Chez nos spéci- 
ments : 1) les côtes 10 sont plus longues que les côtes 9 ; 2) il existe deux petits rameaux supplémentaires sur la côte dorsale en arrière de la naissance des côtes 8 ; 3) pour une taille sensiblement égale à celle de $T$. quintus, la longueur des spicules est presque double.

Il nous paraît donc nécessaire de séparer nos spécimens que nous proposons de nommer Travassostrongylus paraquintus $\mathrm{n}$. $\mathrm{sp}$.

\section{Moennigia sp.}

Matériel: 1 ơ, 2 \%, 1 stade IV.

Localisation: $1 / 3$ antérieur de l'intestin.

Petits Nématodes enroulés selon deux à trois tours de spire senestre le long de leur ligne ventrale. Pore excréteur situé entre l'anneau nerveux et la fin de l'œsophage. Deirides arrondies au même niveau. Glande génitale débutant en avant de la fin de l'œsophage.

Synlophe : Typique du genre Moennigia, mais face dorsale inerme. Les crêtes au nombre de onze ont leur pointe orientée du ventre vers le dos. Le gradient de taille est latéro-ventral (fig. 2, B). Chez la ㅇ, les deux arêtes latérales s'élargissent pour former des ailes de part et d'autre du niveau de la vulve (fig. 2, C, D).

Male : Corps long de $1,2 \mathrm{~mm}$, large de $30 \mu$ dans sa partie moyenne. Vésicule céphalique haute de $40 \mu$ sur $20 \mu$ de large. Anneau nerveux, pore excréteur et deirides situés respectivement à $90 \mu, 120 \mu$ et $123 \mu$ de l'apex. Esophage long de $165 \mu$. Le testicule débute $10 \mu$ en avant de la fin de l'œsophage (fig. 2, A). Bourse caudale typique du genre Moennigia (fig. 2, F). Spicules subégaux longs de $64 \mu$. Leurs extrémités distales paraissent divisées, mais n'ayant qu'un seul spécimen ơ, nous n'avons pu les disséquer, ni observer si le gubernaculum était présent. Cône génital peu marqué, mais papille zéro et papilles 7 bien visibles, en forme de languettes (fig. 2, F).

Femelle: Corps long de $1,35 \mathrm{~mm}$, large de $40 \mu$ dans sa partie moyenne. Vésicule céphalique haute de $42 \mu$ sur $21 \mu$ de large. Anneau nerveux, pore excréteur et deirides situés respectivement à $90 \mu, 140 \mu$ et $148 \mu$ de l'apex. Esophage long de $170 \mu$. L'ovaire débute à $20 \mu$ en avant de la fin de l'œsophage. Il existe deux branches génitales, mais seule l'antérieure est fonctionnelle. Vulve située à $134 \mu$ de la pointe caudale. Vagina vera long de $15 \mu$. La branche génitale postérieure avortée mesure $50 \mu$. L'ovéjecteur antérieur mesure $61 \mu$ (vestibule $21 \mu$, sphincter $10 \mu$, trompe $30 \mu$. Ces mensurations ne sont qu'approximatives, car l'ovéjecteur est fortement dilaté par le passage d'un œuf. La branche utérine antérieure mesure $230 \mu$ et contient trois œufs hauts de $55 \mu$ sur $25 \mu$ de large (fig. 2, E). Queue fine longue de $56 \mu$ avec une pointe caudale de $8 \mu$ (fig. 2, D).

Discussion : Comme nous l'avons vu plus haut, le synlophe et la disposition des côtes bursales sont typiques du genre Moennigia Travassos, 1935. L'absence de crêtes 
cuticulaires sur la face dorsale sépare les spécimens du Metachirops de toutes les espèces de Moennigia dont le synlophe est connu. Parmi les espèces dont le synlophe reste inconnu, la plus proche est $M$. moennigi Travassos, 1935, parasite de Dasypus novemcinctus au Brésil. La disposition des côtes bursales est pratiquement identique. Malheureusement, ne possédant qu'un seul spécimen $\sigma^{\prime}$, nous n'avons pas pu disséquer les spicules, et nous ne pouvons utiliser ce caractère pour la diagnose. De plus, nos

A

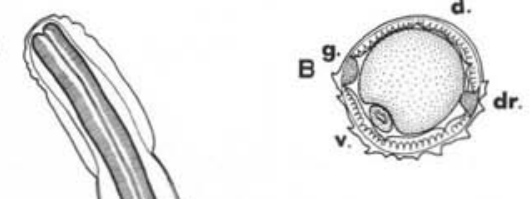

d.

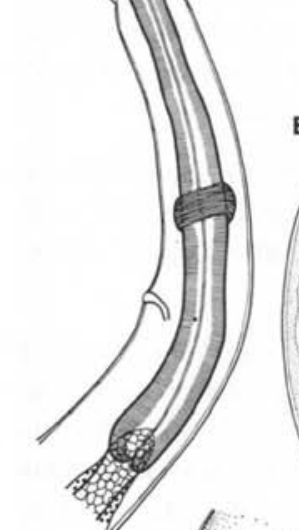

FIG. 2. - Moennigia sp. - A) $\delta$, extrémité antérieure, vue latérale gauche; B) $\delta$, coupe transversale au milieu du corps; C) $\uparrow$, id.; D) $\uparrow$, extrémité postérieure montrant l'élargissement des ailes transversales, vue ventrale; E) $\uparrow$, partie postérieure du corps, vue latérale gauche; F) o', bourse caudale, vue ventrale. 
spécimens sont deux fois plus petits que $M$. moennigi, et le rapport des distances vulve-extrémité caudale sur anus-extrémité caudale est différent (3,1 chez $M$. moennigi, 2,4 chez nos spécimens).

Aussi il nous paraît préférable actuellement, malgré les fortes affinités existant entre les deux espèces, de ne pas identifier nos spécimens à $M$. moennigi .

\section{Viannaia metachirops n. sp.}

MATÉRIEL : 2 б.

LOCALISATION : 2/3 de l'intestin.

Description: Très petits Nématodes fortement enroulés le long de leur ligne ventrale selon 3 à 4 tours de spire.

Corps long de $2,4 \mathrm{~mm}$, large de $45 \mu$ dans sa partie moyenne. Vésicule céphalique haute de $46 \mu$ sur $16 \mu$ de large. Anneau nerveux et pore excréteur situés respectivement à $125 \mu$ et $210 \mu$ de l'apex. Deirides non vues. Esophage long de $190 \mu$ (fig. 3, A).

Bourse caudale asymétrique avec un lobe droit beaucoup plus développé. Disposition des côtes bursales typique du genre Viannaia Travassos, 1914. Spicules fins,
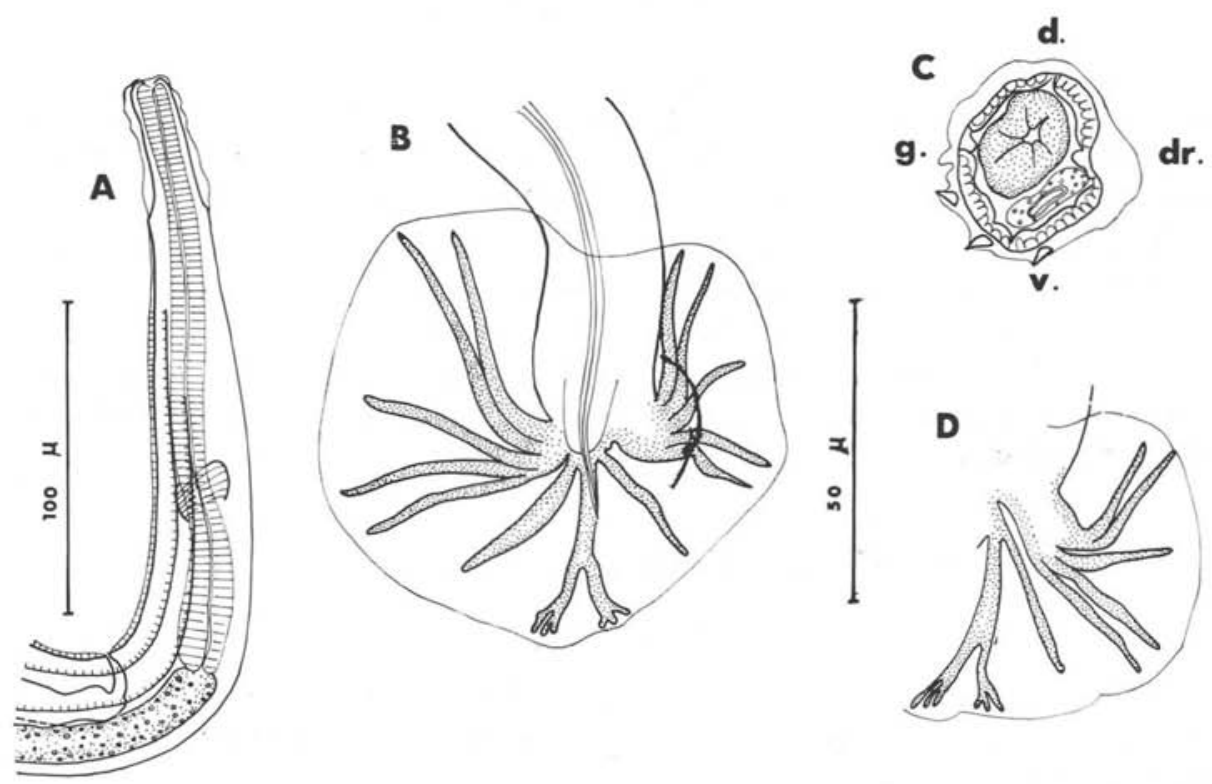

FIG. 3. - Viannaia metachirops n.sp. $\left.-\sigma^{*}-\mathrm{A}\right)$ extrémité antérieure, vue latérale gauche;

B) bourse caudale, vue ventrale; C) coupe transversale au milieu du corps; D) lobe dorsal gauche complètement étalé, vue ventrale. A : éch. $100 \mu ; \mathrm{B}, \mathrm{C}, \mathrm{D}$ : éch. $50 \mu$. 
subégaux, longs de $250 \mu$, à extrémité pointue. Gubernaculum non vu. Cône génital bien développé, mais papilles zéro et 7 non vues (fig. 3, B, D).

Synlophe: Il est formé de trois arêtes cuticulaires ventrales gauches dont la pointe est orientée de la droite vers la gauche. Les arêtes naissent à différents niveaux en arrière de la vésicule céphalique et s'étendent jusqu'au niveau de la bourse caudale (fig. 3, C).

Discussion: Comme nous l'avons vu plus haut, les spécimens du Metachirops présentent les principaux caractères du genre Viannaia Travassos, 1914.

Parmi les espèces du genre, seule $V$. didelphis (Travassos, 1914), parasite de Didelphis au Brésil, possède comme nos spécimens des spicules fins, à extrémité pointue.

Mais, alors que chez cette espèce, les spicules atteignent presque le quart de la longueur du corps, ils ne font que le dixième de celui-ci chez nos spécimens. De plus, ces derniers sont caractérisés par un fort développement des côtes 2 et 3 droites et des côtes 8 plus courtes que la dorsale.

Nous pensons donc qu'il s'agit d'une espèce nouvelle que nous proposons de nommer Viannaia metachirops n. sp.

\section{Numération et répartition des espèces :}

Le tableau ci-contre (fig. 4) récapitule la répartition des espèces dans chacun des tiers de l'intestin. Le résultat est très différent de ce qui a été observé dans des études similaires. Le parasitisme n'est pas très dense et chaque tiers de l'intestin héberge approximativement en nombre égal les deux espèces dominantes.

D'après ces constatations préliminaires, les phénomènes pourraient donc être très différents selon qu'il y a coexistence d'espèces congénères (ce qui est le cas le plus fréquent) ou qu'il $\mathrm{y}$ a coexistence d'espèces appartenant à des genres variés.

L'existence de niches écologiques bien précises qui caractérisent la répartition des espèces dans le premier cas (cf. en particulier Petter, 1966) semble ne pas se manifester dans le second cas.

\section{3. Étude des quatrièmes stades larvaires :}

\section{Viannaia sp.}

Nous avons trouvé un stade IV $q$ dans le deuxième tiers de l'intestin. Cette portion intestinale contenant deux espèces de Viannaia, nous ne pouvons identifier le stade larvaire à l'une des deux.

PRinCIPAles mensurations: Longueur $1,8 \mathrm{~mm}$; largeur dans la partie moyenne du corps $40 \mu$. Anneau nerveux, pore excréteur et deirides situés respectivement à 
$140 \mu, 200 \mu$ et $200 \mu$ de l'apex. Esophage long de $220 \mu$. Emplacement de la vulve : $100 \mu$ de la pointe caudale. Queue longue de $42 \mu$, pointue à l'extrémité (fig. 5, B).

SYNlophe : Il est identique à celui de l'adulte et se compose de trois arêtes ventrales gauches dont la pointe est orientée de la droite vers la gauche (fig. 5, A).

Le genre Viannaia est donc caractérisé par toute absence de transformation ontogénique du synlophe lors du passage du stade larvaire au stade adulte. Ceci nous semble un élément intéressant confirmant, d'une part, le caractère primitif de ce genre (cf. Quentin, 1971), d'autre part, les affinités existant entre les genres Viannaia et Suncinema dont les synlophes sont identiques chez la larve du $4^{\circ}$ stade et chez l'adulte (cf. Durette-Desset, 1974).

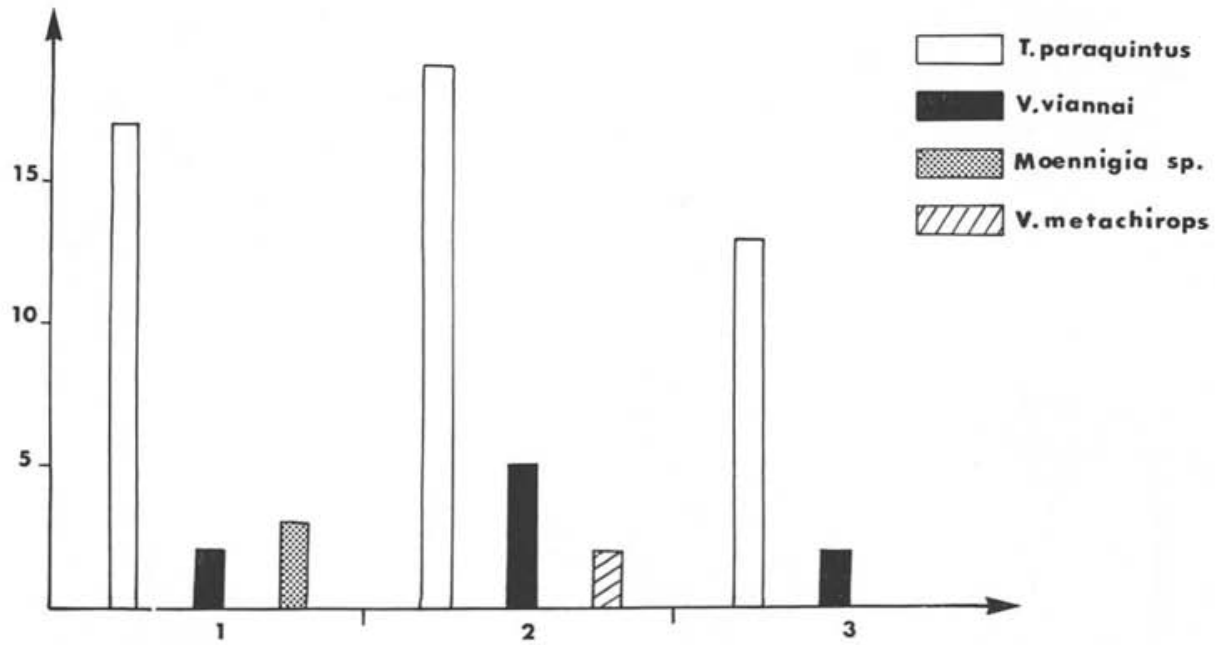

FIG. 4. - Répartition des différentes espèces de Trichostrongyloidea dans l'intestin du Metachirops.

L'intestin est divisé en trois segments. Les chiffres en abscisse indiquent le numéro du segment, depuis l'estomac jusqu'au caecum. La totalité des individus de chaque espèce trouvée dans chaque segment est portée en ordonnée.

\section{Moennigia sp.}

Nous avons trouvé un stade IV $q$ dans le premier tiers de l'intestin.

Principales mensurations: Longueur $1,2 \mathrm{~mm}$, largeur dans la partie moyenne du corps $30 \mu$, anneau nerveux, pore excréteur, deirides situés respectivement à $105 \mu$, $140 \mu$ et $140 \mu$ de l'apex. Esophage long de $165 \mu$. Emplacement de la vulve : $135 \mu$ de la pointe caudale. Queue longue de $58 \mu$ avec une pointe caudale de $8 \mu$ (fig. 5, C).

Synlophe: Il est presque identique à celui de l'adulte, mais n'est formé que de neuf arêtes cuticulaires au lieu de onze, orientées du ventre vers le dos (fig. 5, D). 


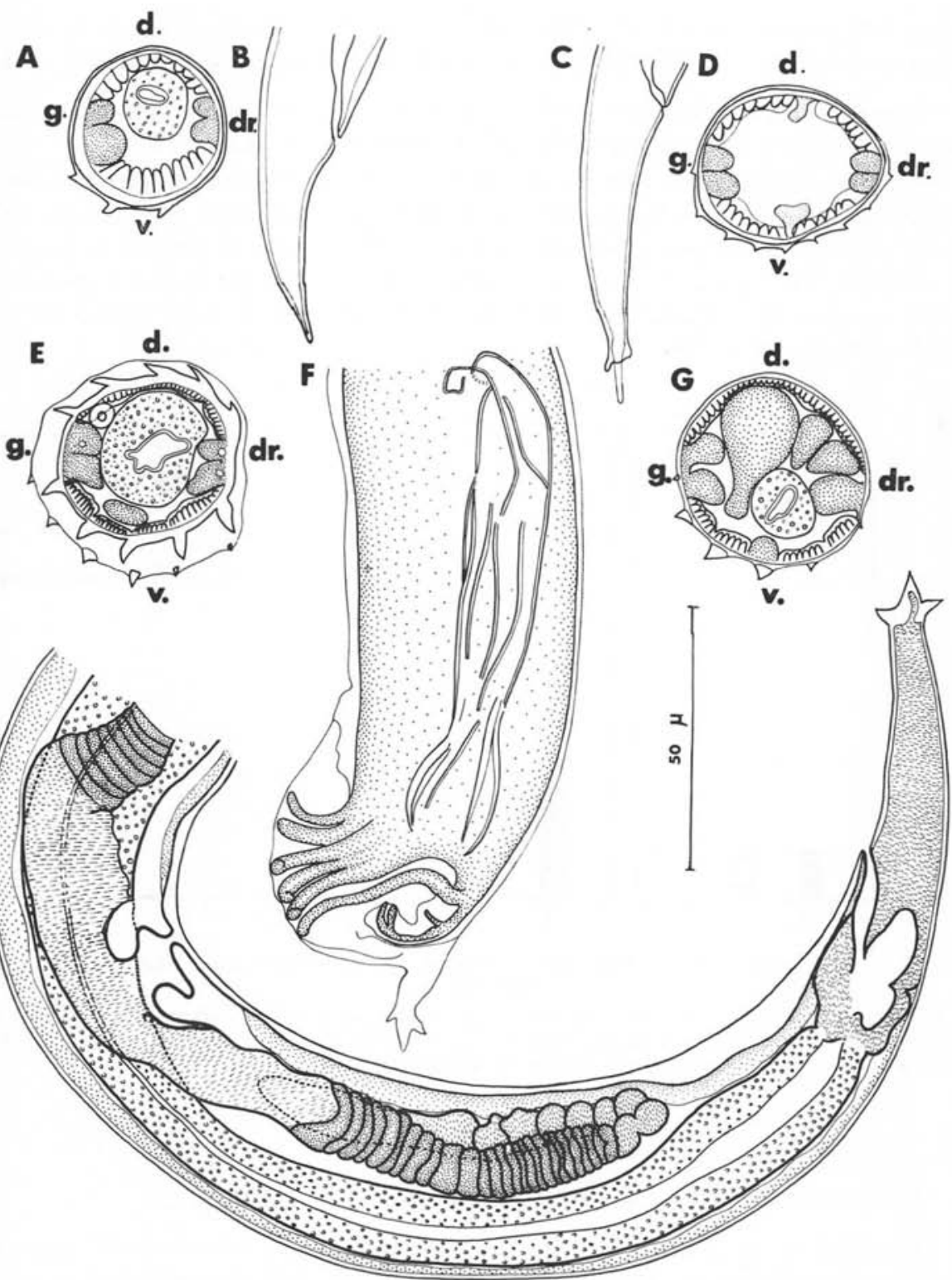

FIG. 5. - Quatrièmes stades larvaires. Viannaia $s p$. $\&$ - A) coupe au milieu du corps; B) extrémité postérieure, vue latérale droite. Moennigia sp. - C) extrémité postérieure, vue latérale droite; D) coupe transversale au milieu du corps. Travassostrongylus paraquintus n. sp. - E) adulte ơ dans la cuticule du $4^{\circ}$ stade larvaire, coupe transversale au milieu du corps; F) id., extrémité postérieure, vue latérale gauche; G) $4^{2}$ stade larvaire + coupe transversale au milieu du corps: $\mathrm{H}$ ) id., extrémité postérieure, vue latérale droite. 
Pour les mêmes raisons que précédemment, nous interprétons l'identité des synlophes de la larve et de l'adulte comme un caractère primitif du genre.

\section{Travassostrongylus paraquintus.}

Nous avons trouvé quatre larves au stade IV dont l'une avec un adulte $\delta$ prêt à muer (fig. 5, E, F), ce qui nous a permis d'identifier l'ensemble à Travassostrongylus paraquintus.

Principales mensurations d'un stade IV $q$ : Longueur $1,85 \mathrm{~mm}$, largeur dans la partie moyenne du corps $40 \mu$. Anneau nerveux, pore excréteur, deirides situés respectivement à $110 \mu, 220 \mu$ et $230 \mu$ de l'apex. Esophage long de $198 \mu$ (92 $\mu$. œophage musculaire, $106 \mu$ œsophage glandulaire). Emplacement de la vulve : $300 \mu$ de la pointe caudale. Queue longue de $72 \mu$ à trois pointes. La pointe caudale de l'adulte, longue de $8 \mu$, est visible à l'intérieur de la cuticule (fig. $5, \mathrm{H}$ ).

SyNLOPHE : Il est formé de six arêtes cuticulaires ventrales dont la pointe est orientée de la droite vers la gauche. Les trois arêtes ventrales adjacentes à l'arête ventrale gauche sont fortement développées (fig. 5, G).

Ce synlophe évoque ceux des genres Viannaia Travassos, 1914 (Heligmosomidae) et Suncinema Durette-Desset, 1973 (Molineinae), mais le nombre d'arêtes cuticulaires est plus élevé.

La connaissance de ce synlophe larvaire nous paraît intéressante à deux points de vue :

1) Elle confirme le fait que le genre Travassostrongylus appartient à la sousfamille des Molineinae (cf. Chabaud, 1959). Nous avons vu en effet que le synlophe est très proche de celui du genre Suncinema, qui appartient à cette sous-famille.

2) Elle confirme nos hypothèses sur l'évolution phylétique des Heligmosomidae : nous avons en effet admis que les arêtes ventrales apparaissaient les premières, puis les arêtes dorsales, et c'est ainsi que nous avons fait dériver le genre Viannella Travassos, 1918, du genre Viannaia, et plus récemment le genre Longistriata Schulz, 1926, du genre Suncinema.

Le fait que le quatrième stade larvaire du genre Travassostrongylus n'a que des arêtes ventrales, alors que l'adulte possède des arêtes sur les deux faces, nous semble apporter une preuve ontogénique aux hypothèses que nous avions formulées.

\section{Bibliographie}

Chabaud (A.-G.), 1959. - Remarques sur la systématique des Nématodes Trichostrongyloidea. Bull. Soc. Zool. de France, 84 (5-6), 473-483.

Duretre-Desset (M.-C.), 1968. - Helminthes de Marsupiaux américains. Trichostrongyloidea. Bull. Soc. Zool. France. 93 (4), 581-594. 
-, 1973. - Nouveaux Nématodes Trichostrongyloidea parasites d'Insectivores Soricidés du Népal; description de Suncinema murini n. gen. n. sp.; forme relique montrant les liens qui unissent les Molineinae et certains Heligmosomes. Bull. Mus., $3^{\circ}$ sér., 136, Zoologie 100, 759-774.

-, 1974. - Sur trois nouveaux Suncinema (Nematoda-Trichostrongylidae) parasites d'Insectivores de Côte-d'Ivoire. (Collection P. Hunkeler). Bull. Mus. (à paraître).

Freitis (T.) et LENT (H.), 1935. - Quelques variations et anomalies bursaires observées chez Viannaia viannai Travassos, 1914, et Viannaia hamata Travassos, 1914. Rev. Med. Cir. Brasil, 43 (9), 253-258.

ORLOFF (I.-W.), 1933. - Sur la reconstruction de la systématique du genre Ostertagia Ransom, 1907. Ann. Parasit. hum. comp., 2 (2), 96-114.

Petter (A.J.), 1966. - Equilibre des espèces dans les populations de Nematodes parasites du colon des Tortues terrestres. Mem. Mus. Nat. Hist. Nat. Nouvelle série. Sér. A, zool., 39 (1), 252 p.

SCHULZ (R.S.), 1926. - Zur Kenntnis der Helminthenfauna der Nagetiere der Union S.S.R. 1. Subordo Strongylata: I. Fam. Trichostrongylidae Leiper, 1912. Trudy Gosudarstv. Inst. Ekseper. vet., 4 (1), 5-32.

Travassos (L.), 1914. - Trichostrongylideos brazileiros (III nota previa). Brazil. Med., 28 (34), 325-327.

-, 1920. - Trichostrongylidae brazileiros. Res Soc. Brasil Sc. (1919) (3), 191-205.

-, 1935. - Contribuçao ao conhecimento dos Trichostrongylidae. Ann. Acad. Brasil Sc.. 7 (4), 355-360. 